\title{
Three-phase Two-level Active Power Factor Correction Circuit Design
}

\section{Ting Zhang, Zuliang Wang and Shiqi Huang}

\author{
Information Engineering College, Xijing University, Shanxi Xian 710123
}

\section{Keywords: Three-Phase Two-Stage; APFC; Boost converter; Buck converter}

\begin{abstract}
The harmonic pollution of power electronic equipment has a strong negative effect on the quality of power supply and power efficiency. In order to improve the power factor, we designed the three-phase two-level active power factor correction (APFC) circuit. The former Boost converter connected with the input power side of the rectifier realized power factor correction (PFC). The post Buck converter has the function of adjusting the output voltage quickly and we designed the corresponding two-level control circuit. We carried out the simulation in the Matlab/simulink software environment. The results showed that the input current waveform is almost the same as that of the bridge rectifier voltage waveform, the input current distortion and harmonics are obviously reduced, and the power factor correction is realized.
\end{abstract}

\section{Introduction}

With the wide spread and application of power electronic devices such as switching power, much more attention has been paid to the problem of PFC. There are two methods can increase the output power efficiency under the same input condition. However, improving power efficiency is limited by the level of power supply circuit which is difficult and the effect is not very obvious. Therefore, improving the power factor is the best choice to increase the output power.

In order to improve the power factor of the converter, a three-phase two-level APFC circuit is proposed. The first level is aiming to improve PFC, and the second level is designed to adjust the output voltage quickly.

\section{Working Principle of the Circuit}

The AC input voltage of the converter is rectified by bridge rectifier, then the DC/DC circuit is followed, the input average current is following the full wave rectified voltage reference value automatically by the reasonable control strategy, at the same time the output voltage is stable. There are two feedback control loop of APFC circuit. One is the input current loop to make DC/DC converter input current waveform and input voltage waveform same, the other is the output voltage loop, aim stabling the DC/DC converter output voltage.

Principle of APFC. The topology of three-phase single switch active power factor correction circuit is single switch Boost converter which is the combination of three-phase bridge rectifier circuit and Boost converter. In a power frequency cycle, the peak value of inductor current which is same to input current is tracking input voltage automat, the circuit is shown in Fig. 1.

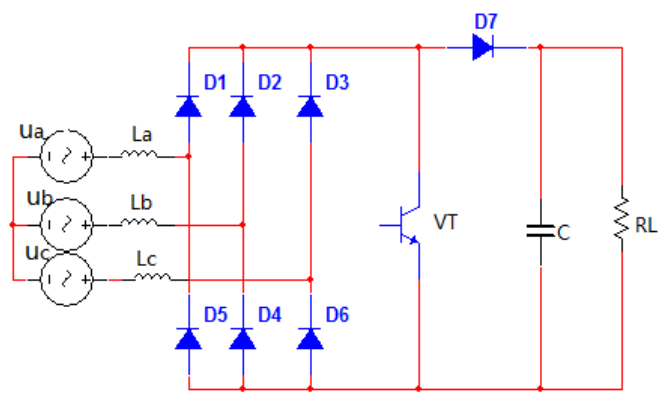

Figure 1. Scheme of two-stage APFC circuit 
The scheme is mainly consist of seven diode, inductor La、 Lb、Lc and capacitor C. D1-D6 is made to rectifier Bridge, while D7 is the freewheeling diode. The value of all the inductors is the same. The controller is mainly to deal with the sample information from the main circuit. It can provide high or low voltage level to control the switch VT work.

Because of three-phase voltage is symmetrical, only analyzing a cycle of ac power can show the works of whole circuit. When the time is $0 \leq \mathrm{t}<\pi / 6$, there will be $u_{c n} \geq u_{a n} \geq 0 \geq-u_{b n}$, and the process works in four stages.

Stage 1: Switch VT is on, input current of each phase increases that is proportional to the phase voltage respectively and the stored energy in inductor increases, capacitor $\mathrm{C}$ provides energy to the load. Because the power voltage absolute value of A phase is most little, so the energy stored is also the least. Stage 2: As the conduction time is constant, so when the switch VT is off each phase current value will be proportional to their respective power supply voltage. When VT is off, both the input power of there-phase and inductor ia $i b$ 、 ic will be supply load with energy. At the same time inductance energy begins to decrease which decided by the input voltage, output voltage and inductance. Stage 3: The switch still be off, the minimum of the absolute value of the phase in the three-phase input current decreases to 0 , namely ia $=0$, at this point in the three-phase rectifier bridge diode D1.Stage 4: The switch still be off,, two phase current of $\mathrm{B}$ and $\mathrm{C}$ also dropped to zero at the same time, all the diodes of three-phase rectifier bridge cutoff, the e load current is provided by the discharge current of the output filter capacitor.

In a switch cycle, the waveform of each phase current is basically a triangle and its peak value is proportional to the power voltage, so the average of each phase current in a switch cycle is half of its peak. While due to the switch frequency is much higher than the grid frequency, the each stage current average can be regarded as instantaneous value, so each phase current will track each phase voltage automatically, and realize power factor correction.

The Three-phase Two-level APFC Circuit. Boost converter only in the switching tube VT cut-off period exists charging current to the output rectifier filter capacitor C. During the switch turn-on, storage capacitor supplies the energy to the load. The voltage of capacitor will drop. Boost converter has the function that increases output voltage, often is used to increase power factor correction. In the Boost circuit, if the load output is not connected, inductive energy storage cannot be consumed which will cause the output voltage rising and cause damage to converter. For Buck converter, inductance $\mathrm{L}$ stores energies during the switch is on. When the switch is off the diode will form release loop.

According to the analysis above, the three-phase two-level APFC circuit uses the Boost converter to achieve power factor correction, uses DC/DC converter to obtain DC output voltage required by the load and adjust the output voltage rapidly, the main circuit is shown in Fig. 2.

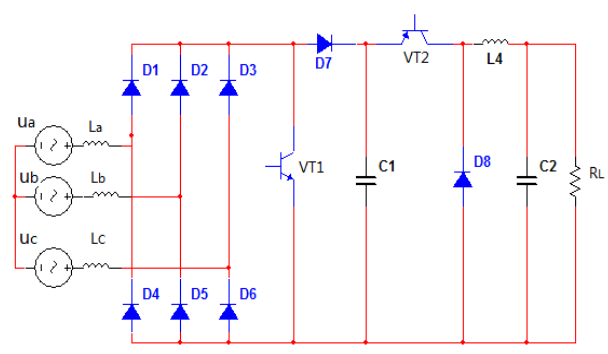

Figure 2. The three-phase two-level APFC circuit

In Fig. 2, the front stage of the three-phase two-level APFC circuit is connected with the input power supply side of rectifying to realize APFC, intermediate is storage capacitor, after class is to make the output voltage stable and ad justly it quickly. The two are independent of each other and have their own switch and control circuit. 
The Design of Control Circuit. In the APFC circuit, the switching circuit of the control circuit must work at high frequency to realize power factor correction. The front single switch Boost converter and latter Buck converter controller both use hysteresis comparator to achieve control. The output voltage of each level is the sampling voltage, namely the input voltage of the hysteresis comparator, by comparing to output high or low level to control switch on or off. The working principle of control part is stated in details.

The switch of former APFC circuit adopts the NPN, the switch turned on when the control level is high, the switch turned off when the control level is low, and the corresponding control circuit adopts a downward hysteresis comparator shown in fig.3. When the input voltage is higher than the upper limit of the downlink hysteresis comparator threshold voltage, the comparator outputs low level, when the input voltage is lower than the lower limit of the downlink hysteresis comparator threshold voltage, the comparator outputs high output, so as to control the NPN transistor action.

The switch of latter DC/DC converter uses PNP transistor, the switch is off when the control level is high, the switch turned on when the level is low, the corresponding control circuit uses the upstream hysteresis comparator shown in Fig. 4.

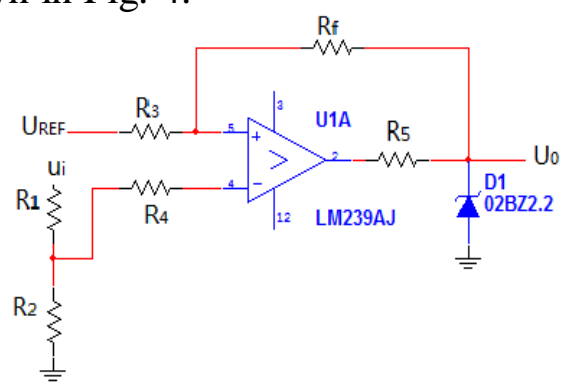

Figure 3. Control circuit of NMOS

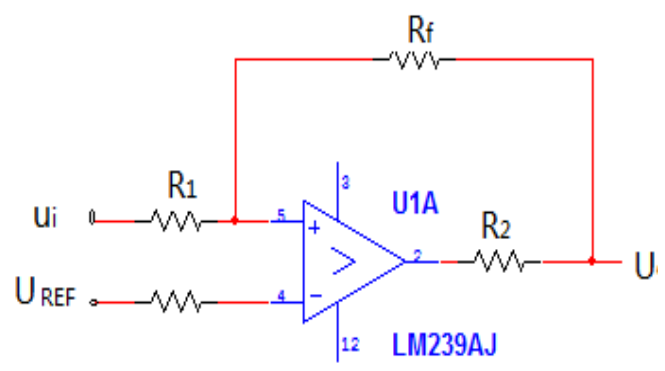

Figure 4. Control circuit of PMOS

When the input voltage of the uplink hysteresis comparator is higher than the upper limit threshold voltage, the comparator outputs a high level. The input voltage of the uplink hysteresis comparator is lower than the lower limit threshold voltage, the comparator outputs low level, so as to control the PNP transistor to act.

\section{Simulation Result}

In the Simulink environment, the three-phase two-level active power factor correction circuit is simulated, and the experimental waveforms are obtained. The simulation parameters are as follows: AC input voltage: three phase voltage $(9 \sim 12 \mathrm{~V})$, the first output DC voltage: $20 \mathrm{~V}$, after the output DC voltage: $10 \mathrm{~V}$, switching frequency: $50 \mathrm{kHz}$.

The Switch Waveform. The three-phase two-level APFC circuit is composed of two independent converters, each level two has their own switch and control circuit. The switch turn-on and turn off 
periodic in a input voltage period to achieve the control effect, the measured base switch waveform as shown in Fig. 5.

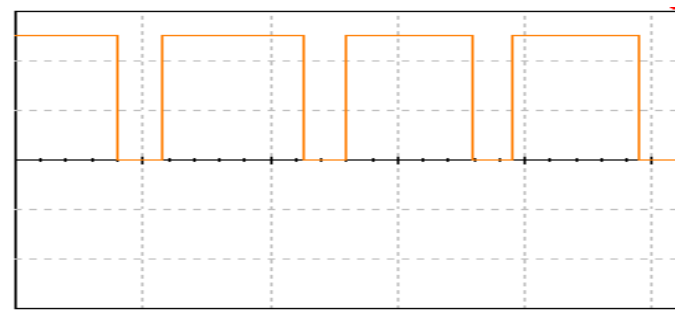

a) Switch waveform of Boost convert

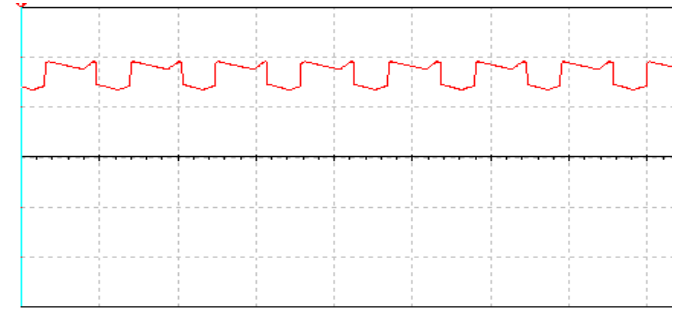

b) Switch waveform of Buck converter

Figure 5. control waveform of switch

Fig. 5a) each small grid in the oscilloscope is $2 \mathrm{~V}$, hysteresis comparator output is high, the voltage is $5 \mathrm{~V}$, control NPN switch tube conduction, the output voltage is 0 , the control switch turn off. Fig. 5b) each small grid in the oscilloscope is $10 \mathrm{~V}$, hysteresis comparator output is high, the voltage is about $20 \mathrm{~V}$, control PNP switch off, the output voltage is about $13 \mathrm{~V}$, the control switch conduction.

Waveform of Input Voltage and Current. The input voltage and current waveforms of the three-phase two-level APFC circuit is obtained by simulation are shown in Fig. 6.

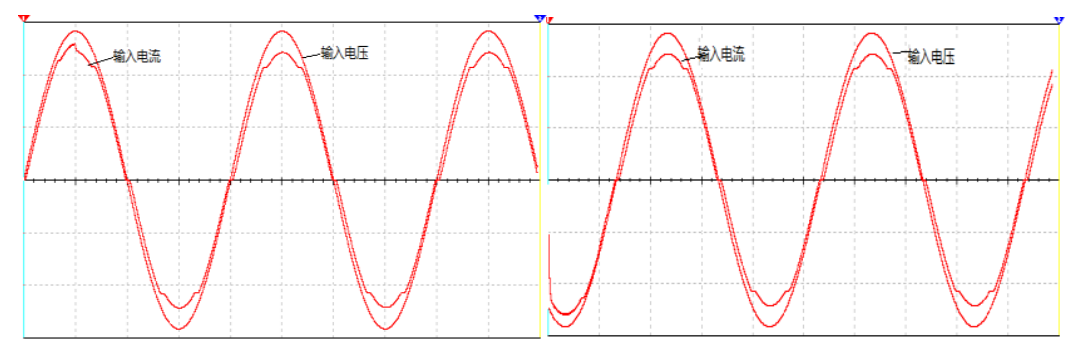

a) Waveform of the first phase

b) Waveforms of second phase

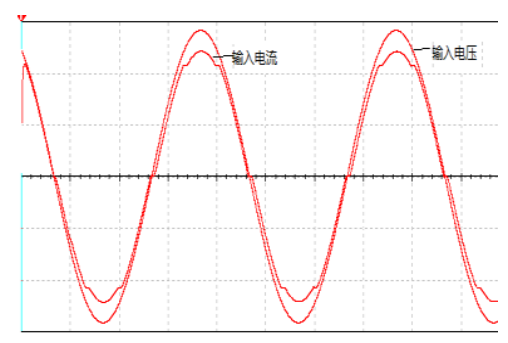

c) Waveforms of the third phase

Figure 6. Waveform of input voltage and current

It can be seen from the simulation results that the phase difference between the three-phase input current and the input voltage is 0 , and the waveform is close to the sine wave, which is consistent with the theory, also proves that the scheme is correct and feasible.

\section{Conclusion}

The three-phase two-level APFC circuit consists of the Boost circuit and Buck converter, and the Boost circuit realizes power factor correction (PFC), the Buck converter can adjust output voltage fast. Downlink hysteresis comparators are the control circuits. The simulation results show that the input current waveform of Boost converter and bridge rectifier voltage waveforms are almost the same, the power factor correction function is achieved.

\section{Acknowledgements}

It is supported by the Research Fund Project of Xijing University (XJ160228).

\section{References:}

[1] Y.Q Pei, X Yang, Design and Application of Correction Circuit in Switching Power Supply Power Factor [M].Beijing: China Machine Press, 2010. 
[2] C.Q Du, Z.Q Pan, etc. Research on Charging System of Electric Vehicle [J].Chinese Journal of Power Sources, 2016, (06):1277-1279+1329.

[3] L Liu, Z.Q He, Application Research of APFC in Charging-Pile [J].Industrial Control Computer, 2016, (09):128-129.

[4] Z.C Sun, W.X Wang and J.W Tian. APFC Correction Method of A Three-Phase Controlled Rectifier Circuit [J].Microcomputer and its Applications, 2016, (20):27-30.

[5] L.H Yang, H.Q Ben, and so on. Research on Suppression Strategies of APFC Current Harmonics with Three Phase Single Stage Full Bridge Type [J].Power Electronics, 2016,(02):60-62.

[6] E. Blank,G. Welzel, M. Sütterlin, F. Wenz. Quality of Life after Intraoperative Radiotherapy (IORT) as a Boost in Patients with Early Breast Cancer [J]. Geburtsh Frauenheilk, 2010, 70(10): 22-25.

[7] C Yang, X.C Kong, etc. Communication Design of Power Line Communication in Shearer Based on Embedded OFDM Technology [J].Journal of Jixi University,2016,(06):46-48.

[8] W.W Zhang, Z.L Liu, etc. Switching Power Supply Design with APFC Function of AC-DC [J].Electronic Measurement Technology, 2016, (05):22-26.

[9] J Hui, H.J Fu, Research and Design of Non-Destructive Step Up APFC Circuit [J].Power Electronics, 2016, (05): 62-64.

[10]Z.X Wang, Y.G Wei, Study on Single-Stage AC/DC Conversion Device Based on Single-Cycle Control[J]. Recent Advances in Electrical and amp; Electronic Engineering, 2016, 9(1): 3-7. 